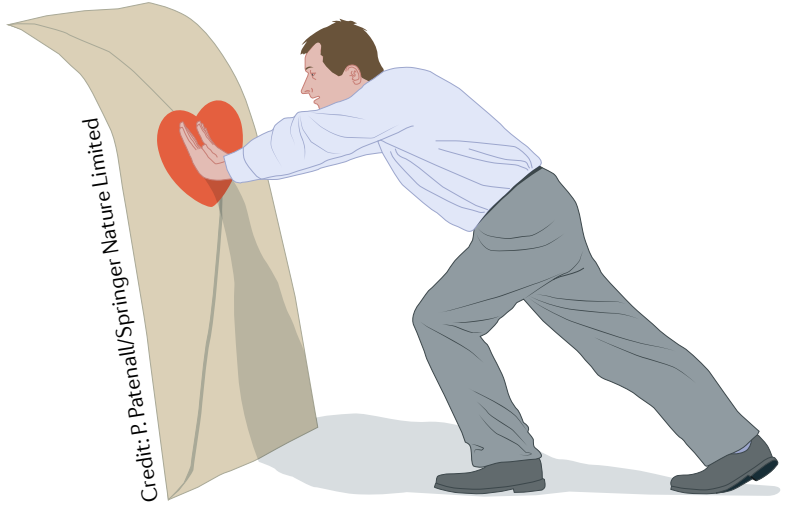

in 25 patients in the envelope group (a relative risk reduction of $40 \%$ ).

Patients in the envelope group were not at higher risk of procedure-related or system-related complications than those in the control group.

"This study provides comprehensive data on CIED

...prevention has been the cornerstone to address the issue of CIED infection infection and strong evidence for the use of the antibacterial envelope for infection prevention in this patient population," concludes Tarakji.

ORIGINAL ARTICLE Tarakji, K. G. et al. Antibacterial envelope to prevent cardiac implantable device infection. N. Engl.J. Med. https://doi.org/10.1056/NEJMoa1901111 (2019)

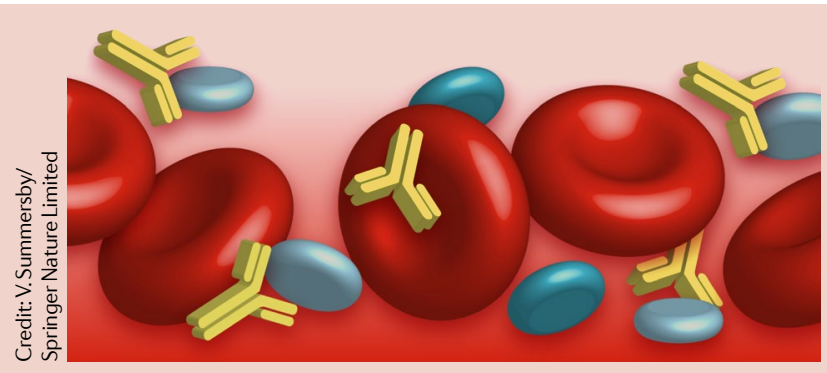

Ticagrelor reversal occurred within 5 min after PB2452 initiation, and persisted for $>20 \mathrm{~h}$. No evidence of a rebound in platelet activity after drug cessation was observed. "Hopefully, these data will ultimately help to get the drug approved for use," says Bhatt. "Future studies will need to include patients with actual bleeding complications or in need of urgent or emergency surgery."

Karina Huynh

occurred within 5 min after PB2452 initiation based ticagrelor reversal agent in healthy volunteers. N. Engl.J.Med. https://doi.org/ 10.1056/NEJMoa1901778 (2019) FURTHER READING Levy, J. H. et al. Reversal agents for non-vitamin $\mathrm{K}$ antagonist oral anticoagulants. Nat. Rev. Cardiol. 15, 273-281 (2018)
Isobel Leake

ANTITHROMBOTIC THERAPY

\section{Tailoring antithrombotic strategies for high-risk AF populations}

Patients with atrial fibrillation (AF) and a recent acute coronary syndrome (ACS) or who have undergone percutaneous coronary intervention $(\mathrm{PCl})$ often receive both antiplatelet therapy (to prevent ischaemic events and stent thrombosis) and oral anticoagulants (to prevent stroke and systemic

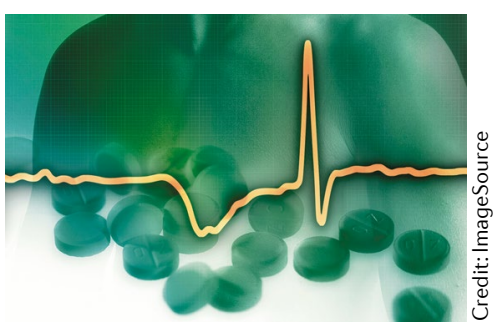
embolism). However, how to select the best antithrombotic regimen for this patient population to minimize the risk of bleeding without compromising protection is an important unanswered question. Now, results from the AUGUSTUS trial, presented at ACC.19, indicate that in this high-risk group of patients, a regimen that includes a $\mathrm{P}_{2} \mathrm{Y}_{12}$ inhibitor plus apixaban, at the dose recommended for stroke prevention, without aspirin results in less bleeding and fewer hospitalizations, without significant differences in ischaemic events, than the use of regimens that include a vitamin K antagonist (VKA), aspirin or both.

The trial had a two-by-two factorial design to assess the independent effect of direct-acting anticoagulants and antiplatelet therapy in patients with nonvalvular $\mathrm{AF}$ and a recent $\mathrm{ACS}$ or $\mathrm{PCl}$ and who were planning to take a $\mathrm{P} 2 \mathrm{Y}_{12}$ inhibitor. A total of 4,614 patients were randomly assigned to receive apixaban or a VKA and to receive aspirin or placebo for 6 months. No significant interaction was observed between the two randomization factors, which allowed the independent analysis of the two main comparisons.

Apixaban was both noninferior and significantly superior to VKA for the primary outcome of major or clinically relevant nonmajor bleeding. The primary outcome occurred in $10.5 \%$ of patients in the apixaban group and in $14.7 \%$ of those in the VKA group, a $31 \%$ reduction in the relative risk of bleeding with apixaban (HR $0.69,95 \% \mathrm{Cl} 0.58-0.81, P<0.001$ for both noninferiority and superiority). Analysis of the secondary outcomes indicated a $17 \%$ reduction with apixaban in the relative risk of death or hospitalization compared with the use of a VKA, which was primarily driven by a reduction in all-cause hospitalization. The incidence of the composite of death and ischaemic events was similar in both groups.

The use of aspirin significantly increased the relative risk of bleeding by $89 \%$ compared with placebo ( $\mathrm{HR} 1.89,95 \% \mathrm{Cl} 1.59-2.24, P<0.001)$. The rates of death or hospitalization were similar in the aspirin and placebo groups, as were the rates of the composite ischaemic events. Of note, avoiding aspirin resulted in a non-significantly higher incidence of coronary ischaemic events, but event rates were low, and the trial was not powered to assess differences in individual ischaemic outcomes.

"For the majority of patients, if you look at the totality of data, one lesson learned that is now confirmed by AUGUSTUS is that less is more," highlights Renato Lopes, lead investigator of the trial. "If we use a P2Y $\mathrm{Y}_{12}$ inhibitor with one of the novel oral anticoagulants at the right dose for stroke prevention in $\mathrm{AF}$ - in this case, apixaban $5 \mathrm{mg}$ twice daily - you have the safest strategy and you don't seem to pay a high cost on ischaemic events for omitting aspirin," he explains, and remarks that future studies assessing different durations of antithrombotic regimens might further refine the treatment of these high-risk patients.

Irene Fernández-Ruiz 